\title{
Quantifying the Risk of Coronary Artery Disease in a Community. The Bambuí Project
}

\author{
Sandhi Maria Barreto, Valéria Maria Azeredo Passos, Alisson Renê Aquino Cardoso, \\ Maria Fernanda Lima-Costa
}

Belo Horizonte, MG - Brazil

\begin{abstract}
Objective - To determine the coronary risk profile in adults and elderly in a community.

Methods - The study comprised a sample of adults (30-59 years, $n=547)$ and the entire elderly population $(60-74$ years, $n=1165)$ residing in Bambui town, Brazil. The Framingham score based on sex, age, smoking, diabetes mellitus, systolic and diastolic blood pressure, total cholesterol, and $H D L-C$ was used. The score based on age and sex was defined as "expected" and compared with the mean score obtained by the sum of all risk factors in each age group and sex (score "observed").
\end{abstract}

Results - The difference between the scores "observed" and "expected" increased with aging in both sexes. Smoking increased the difference from 30 years of age onwards, in both sexes, and hypertension was important in men above the age of 30 years and in women above the age of 50 years. Diabetes and elevated total cholesterol increased the risk of the disease above the age of 50 years in both sexes. A higher level of $H D L-C$ reduced the risk among men above the age of 30 years, with no significant difference among women. Less schooling $(<4$ years versus $\geq 4$ years) was associated with a higher score in adults of both sexes, but not among the elderly.

Conclusion - Based on these results, in the community studied, the risk of coronary artery disease may be reduced up to $44 \%$ in men and $38 \%$ in women.

Key words: coronary artery disease, risk score, population-based study

Faculdade de Medicina da UFMG and Centro de Pesquisas René Rachou of the Fundação Oswaldo Cruz

Mailing address: Sandhi Maria Barreto - Laboratório de Epidemiologia e Antropologia Médica - Av. Augusto de Lima, 1715 - CP 1743 - Cep 30190-002 Belo Horizonte, MG, Brazil

Received 10/14/02

Accepted 3/10/03

English version by Stela Maris C. e Gandour
Cardiovascular diseases account for 18 million deaths per year in the world, coronary artery diseases and cerebrovascular diseases being responsible for two thirds of these deaths and for approximately $22 \%$ of the 55 million deaths due to all causes ${ }^{1}$. Estimates on mortality due to cardiovascular diseases according to the region indicate that developing countries contribute with a greater part of the overall burden of mortality due to the disease than developed countries $^{2}$, with a relative excess of $70 \%{ }^{3}$.

In addition to being an important cause of mortality, cardiovascular diseases also represent a great burden in regard to morbidity, being at the top of the list of causes of years lived with disability (DALY - Disability Adjusted Life Years Lost). DALY is a measure developed by the Global Burden of Disease Study, which comprises the sum of the life years lost to early mortality and years lived with disability, adjusted to the severity of the diseases ${ }^{4,5}$. In countries like Brazil, the increase in the elderly population and the increase in longevity associated with changes in diet pattern and lifestyle have a strong impact on the morbidity and mortality pattern. It is estimated that in 2020 , three quarters of the deaths occurring in developing countries will be related to population aging, most of these deaths being due to chronic diseases, especially to diseases of the circulatory system, cancer, and diabetes mellitus ${ }^{6}$.

Despite this evidence, epidemiological studies have shown that cardiovascular diseases are a relatively rare cause of death in the absence of major risk factors ${ }^{1}$. Almost $75 \%$ of the new cases of cardiovascular diseases occurring in developed countries in the 1970s and 1980s could be explained by inadequate diet and physical activity, expressed by high lipid levels, obesity, and increased blood pressure, associated with smoking ${ }^{7}$. The study of these risk factors relates their presence and intensity to the development of the disease. These prospective studies were responsible for the development of risk scores, which allow for the estimation of the probability of developing a certain cardiovascular disease in a defined time interval. These risk scores, in addition to being useful in foretelling a cardiovascular event, aid in its prevention and in the reduction in its incidence in individuals and populations ${ }^{7-9}$. 
Currently in Brazil, cardiovascular diseases account for $33 \%$ of the deaths with known causes. Between 1996 and 1999 , they were the first cause of hospitalization in the public sector among individuals aged 40 to 59 years $(17 \%)^{10}$ and for those aged 60 years or more $(29 \%)^{11}$. In the municipality of Bambuí in the state of Minas Gerais, Brazil, cardiovascular diseases (ICD-9: 390-459) also topped the list of causes of death in 1991 (34\%) and were the second cause of hospitalization in the public sector in $1993(18.5 \%)^{12}$. Although the transmission of $T$. cruz $i$ has been interrupted for approximately 20 years, the prevalence of infection in that community remains elevated due to the cohort effect ${ }^{13}$.

The present study aimed at disclosing the risk profile of coronary artery disease in Bambuí by estimating the risk of developing the disease within 10 years ${ }^{14}$. In addition, this study assesses the contribution of each risk factor included in this algorithm to the final probability of developing coronary artery disease, as well as the influence of schooling, familial income, and the presence of Trypanosoma cruzi infection.

\section{Methods}

The present study is part of the Bambuí Project, a longitudinal study on aging ${ }^{12}$. A simple probabilistic sample of the population aged 30 to 59 years and the entire population aged 60 to 74 years living in the municipality of Bambuí were selected. The size of the adult sample (18-59 years) was sufficient to estimate the prevalence of risk factors $=0.50$, with a confidence interval of 0.95 , losses of 0.20 , and accuracy of 0.03 . The interviews, physical measurements, and blood tests were carried out between October 1996 and August $1997^{12}$.

The parameters used for constructing the risk score were as follows: sex, age, smoking, diabetes mellitus, systolic and diastolic blood pressure, total cholesterol, and HDL-C.

Blood pressure measurements were taken with the individuals seated after resting for at least 15 minutes, their arm level with the heart, and with no caffeine ingestion or smoking in the preceding 30 minutes. Three measurements were taken at intervals of at least 2 minutes. The first blood pressure measurement was disregarded, the mean of the last 2 measurements being considered for analysis purpose ${ }^{15}$. The construction of the score was based on the most elevated systolic or diastolic blood pressure value, when these measurements were within a distinct range of scores, using as a reference the classification of the $\mathrm{V}$ Joint Committee on Detection, Evaluation and Treatment of High Blood Pressure (1993), as previously reported ${ }^{15}$.

For the laboratory tests, blood was collected after a 12hour fasting period. The levels of total cholesterol, HDL-C, and LDL-C were defined according to the criteria recommended by the National Cholesterol Education Program (1993).

Individuals who had smoked at least 100 cigarettes throughout their lives and who continued smoking during the period of the interview were considered smokers. Individuals being treated with insulin or oral antidiabetic drugs, or those with high glycemic levels $(\geq 126 \mathrm{mg} / \mathrm{dL})$, were classified as having diabetes mellitus ${ }^{14}$.
Risk of coronary artery disease in a community. The Bambuí project
The presence of antibodies against $T$. cruzi was detected through the indirect hemagglutination (Biolab) and ELISA (Abbott Brasil) tests. Infection with T. cruzi was defined as a positive serology (levels $\geq 1: 80$, in indirect hemagglutination, and ratio between absorbance of the sample and cut-off of the reaction $>1.1$, in the ELISA test) in both tests ${ }^{12}$.

In the interview, all individuals reporting a previous medical diagnosis of myocardial infarction $(n=66)$ were excluded from the calculation of the score for coronary artery disease ${ }^{13}$

The scoring and the final probability of developing coronary artery disease within 10 years proposed by Wilson et al ${ }^{14}$ were used (Chart I). The score based on age for each sex was defined as the score "expected" and was calculated based on an individual at the same age, with normal blood pressure, levels of total cholesterol between 160 and $199 \mathrm{mg} / \mathrm{dL}, \mathrm{HDL}-\mathrm{C} \geq 45 \mathrm{mg} / \mathrm{dL}$ for men and $\geq 55 \mathrm{mg} / \mathrm{dL}$ for women, nonsmokers and nondiabetics. The score "observed" was calculated by adding the points of all factors composing the risk profile for men and women. For each age, the mean and median of the score observed was calculated, the former being maintained because it did not differ from the latter. The score expected was then compared with the mean score observed at each age. Then, the contribution of each isolated factor for the difference between the score observed with the addition of each factor and the score expected based on age and sex was analyzed.

The individuals were classified according to their risk range and the score observed. For this, the individuals with a previous history of myocardial infarction were classified as those with the greatest risk, because previous myocardial infarction increases the risk of a new cardiovascular event ${ }^{16}$. The 12 individuals, for whom this information was not available were considered for study purposes as having a negative previous history for myocardial infarction.

Finally, the medians of the global scores of the adult (30-59 years) and elderly (60-74 years) participants of both sexes were compared according to schooling $(<4$ years vs $=$ 4 years of schooling) and income ( $<2$ minimum wages vs $=2$ minimum wages per month). Considering the high prevalence of Chagas' disease in the municipality, particularly

\begin{tabular}{|c|c|c|c|c|}
\hline \multicolumn{5}{|c|}{$\begin{array}{l}\text { Chart I - Example of the construction of the risk score for coronary } \\
\text { artery disease in the time period of } 10 \text { years for a man and a woman } \\
\text { with the same ages and lifestyle }\end{array}$} \\
\hline Risk factors & & \multicolumn{3}{|c|}{ Points * } \\
\hline Age & (years) & 55 & 4 & 7 \\
\hline Total cholesterol & $(\mathrm{mg} \%)$ & 250 & 2 & 1 \\
\hline HDL-C & $(\mathrm{mg} \%)$ & 39 & 1 & 2 \\
\hline Blood pressure & $(\mathrm{mmHg})$ & $146 / 88$ & 2 & 2 \\
\hline Diabetes & (yes/no) & Yes & 2 & 4 \\
\hline Smoking & (yes/no) & No & 0 & 0 \\
\hline Score & & & 11 & 16 \\
\hline Estimated risk $*$ & & & $31 \%$ & $24 \%$ \\
\hline
\end{tabular}


among the elderly ${ }^{13}$, the risk profile of this population was analyzed according to the presence of infection by $T$. cruzi. The statistical significance was tested using the Wilcoxon rank sum test to compare the medians. Data were processed and analyzed using Stata Statistical software, version $1.7^{17}$.

This study was carried out according to the current guidelines for the development of health research in Brazil and was approved by the Committee on Ethics of the Fundação Oswaldo Cruz. Participation in the study was voluntary, and written informed consent was obtained prior to the interviews and the medical and laboratory examinations ${ }^{12}$.

\section{Results}

This study comprised 1712 individuals, 547 adults (82.5\% of the eligible population) and $1165(85.9 \%)$ elderly. Table I shows the distribution of the individuals included in the study according to age group, sex, and the prevalence of risk factors. The 2 most prevalent risk factors in adult men (30-59 years) were low levels of HDL-C and smoking, and those in the elderly (60-74 years) were the reduced levels of HDL-C and high blood pressure. Adult women had a lower prevalence of all risk factors as compared with adult men. Elderly women had a higher prevalence of high levels of total cholesterol and diabetes as compared with that of elderly men. Smoking was the only risk factor with a lower prevalence among the elderly as compared with that in adults, of both sexes, the reduction being more significant among women.

The results show that the mean global score observed in each age group and sex and comprising the modifiable risk factors grows gradually apart from the mean score expected based only on age and sex (fig. 1). Among men, the difference between the score expected and the mean score observed increased according to the age group of the individual, while among women the difference between the expected and observed scores significantly increased from the age of 50 years onwards.

The probability of developing coronary artery disease within 10 years obtained from the global risk score for men

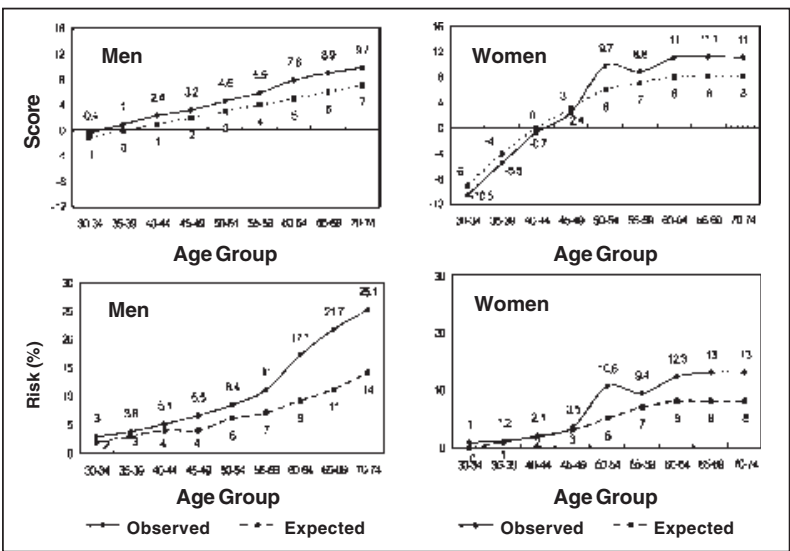

Fig. 1 - Global and expected Framingham risk score and global and expected risk of developing coronary artery disease in 10 years according to age and sex in a population aged 30 to 74 years. Bambuí Project, 1997

and women is shown in figure 1 . The differences in the estimates of the mean global risk and the expected risk based on age are greater among men aged 55 years or above, and these differences increase as age advances until reaching the greatest difference in the oldest age group (70-74 years). Among men, the greatest mean risk observed was $25 \%$ and the greatest mean risk expected was $14 \%$. Among women aged 50 to 54 years, a twofold increase occurred in the difference between the estimates of mean global and expected risks, with a tendency towards maintenance from then on. Among women, the greatest mean risk observed was 13\% and the greatest mean risk expected was $8 \%$.

Analyzing each risk factor alone (figs. 2 and 3), elevated blood pressure was observed to have contributed to increasing the difference between the score expected and that observed among men aged 30 years or more, and its importance increased with age. Among women, elevated blood pressure contributed to reducing the mean score observed in the lowest age groups and to increasing that difference between 50 and 60 years.

The presence of high levels of total cholesterol has

Table I - Prevalence of risk factors for coronary artery disease among men and women aged 30 to 59 years and 60 to 74 years. Bambuí Project, 1997

\begin{tabular}{|c|c|c|c|}
\hline & $\mathrm{CI}(95 \%)$ & $\%$ & \\
\hline Risk factors & $30-59$ anos & 60-74 years & $\chi^{2}, \mathrm{p}$ \\
\hline & MEN & & \\
\hline Cholesterol $\geq 240 \mathrm{mg} / \mathrm{dL}$ & $13.6(13.64-13.68)$ & 30 & $27.7 \mathrm{p}<0.001$ \\
\hline HDL Cholesterol $\leq 45 \mathrm{mg} / \mathrm{dL}$ & $46.5(46.44-46.56)$ & 57.5 & $5.5 \mathrm{p}=0.02$ \\
\hline Blood pressure $\geq 140 / 90 \mathrm{mmHg}$ & $20.2(20.15-20.25)$ & 47.5 & $48.8 \mathrm{p}<0.001$ \\
\hline Smoker & $39.9(39.84-39.96)$ & 30.6 & $5.3 \mathrm{p}=0.02$ \\
\hline \multirow[t]{2}{*}{ Diabetes mellitus } & $3.5(3.48-3.52)$ & 13.5 & $16.8 \mathrm{p}<0.001$ \\
\hline & WOMEN & & \\
\hline Cholesterol $\geq 240 \mathrm{mg} / \mathrm{dL}$ & $12.2(12.16-12.24)$ & 48.7 & $133.7 \mathrm{p}<0.001$ \\
\hline HDL Cholesterol $\leq 45 \mathrm{mg} / \mathrm{dL}$ & $35.4(35.35-35.45)$ & 43 & $5.3 \mathrm{p}=0.02$ \\
\hline Blood pressure $\geq 140 / 90 \mathrm{mmHg}$ & $16(15.96-16.04)$ & 42.5 & $47.9 \mathrm{p}<0.001$ \\
\hline Smoker & $24.1(23.95-24.15)$ & 11.5 & $35.1 \mathrm{p}<0.001$ \\
\hline Diabetes mellitus & $3.1(3.08-3.12)$ & 15.9 & $35.7 \mathrm{p}<0.001$ \\
\hline
\end{tabular}




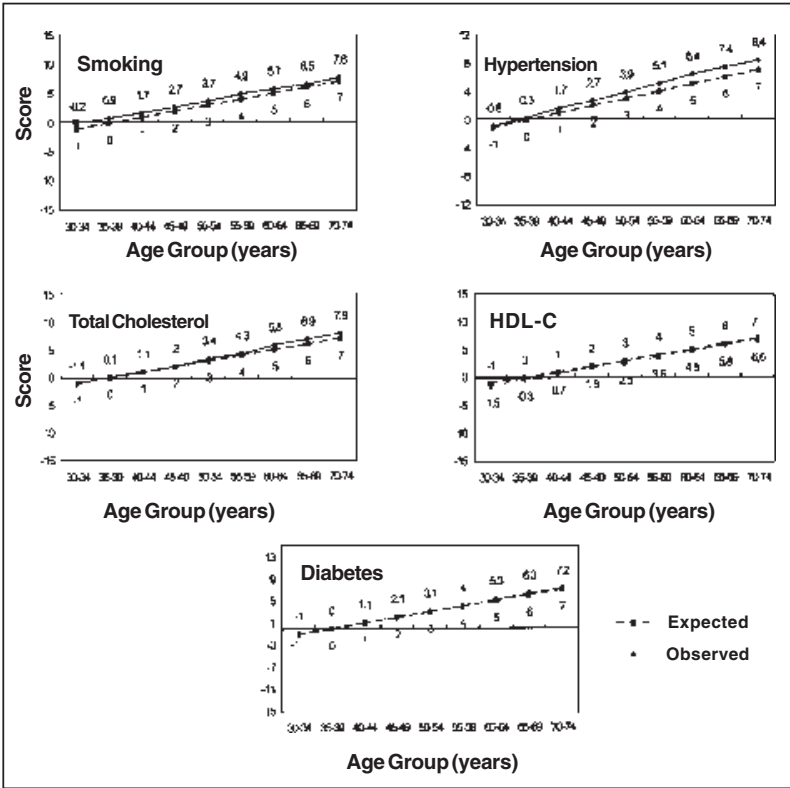

Fig. 2 -Expected Framingham risk score for coronary artery disease based on age and sex and observed score considering the contribution of each risk factor alone in men. Bambuí Project, 1997.

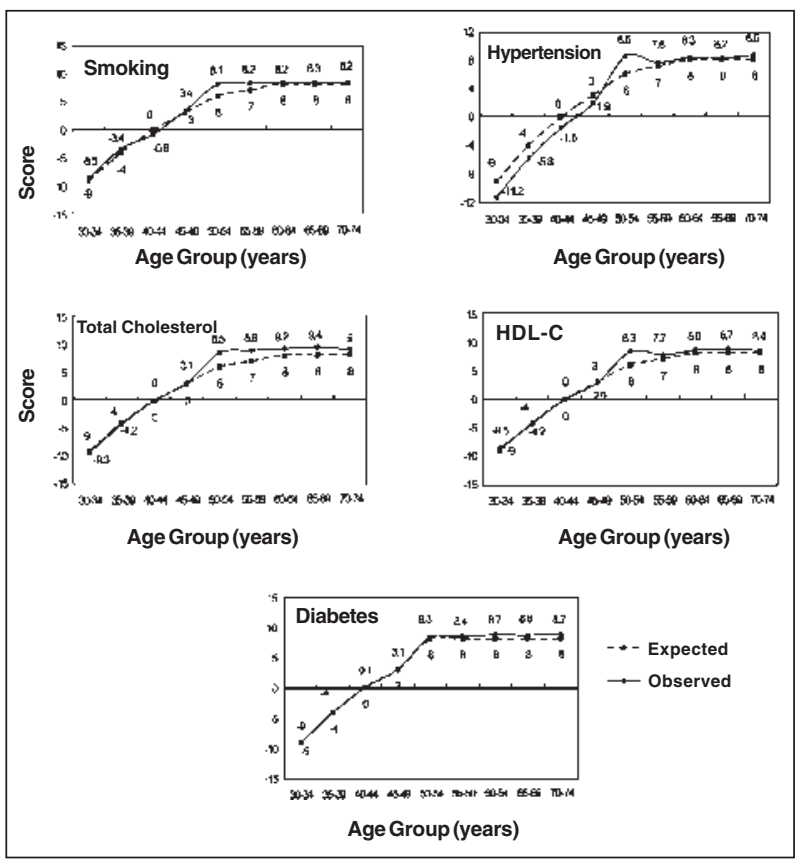

Fig. 3 - Expected Framingham risk score for coronary artery disease based on age and sex and observed score considering the contribution of each risk factor alone in women. Bambuí Project, 1997.

contributed to increasing the difference between the score expected and the mean score observed in men aged 50 years and above and in women aged 45 years and above.

Smoking was also an important factor in the difference between the score expected and the mean score observed in men aged 30 years and above, with this difference being about the same in all ages. Among women, smoking began to contribute to increasing this difference from the 45 to the 65 years.

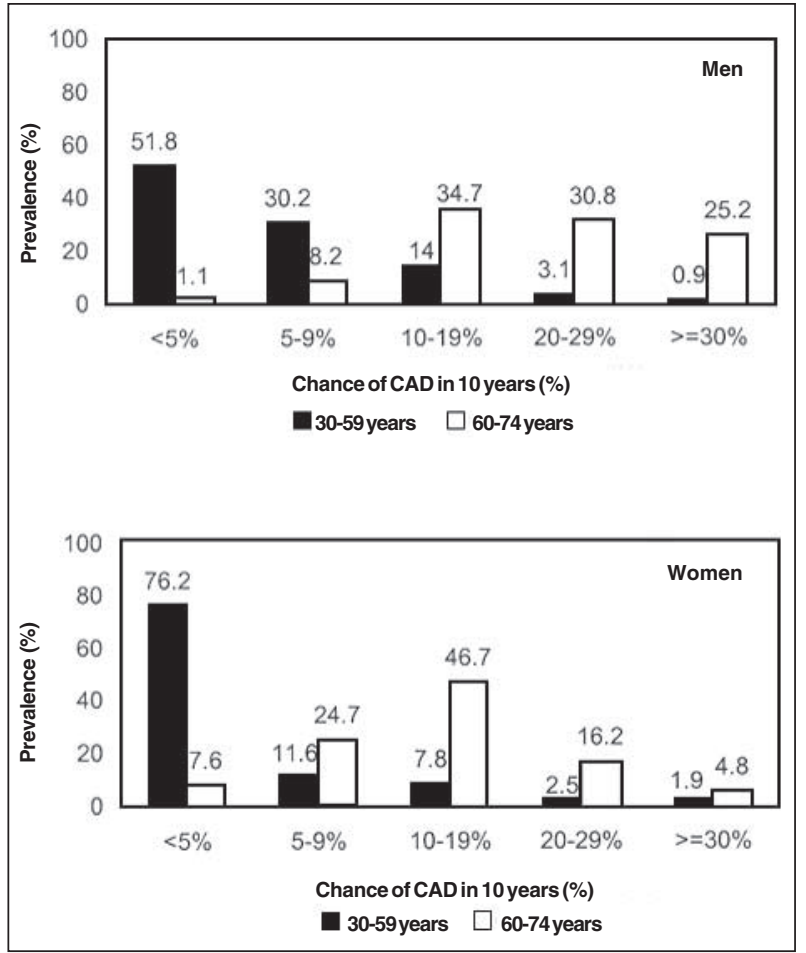

Fig. 4 - Distribution of the individuals according to the probability of developing coronary artery disease within 10 years by age group and sex using the Framingham score. Bambuí Project. Note: Individuals with a previous history of infarction $(n=66)$ were included in the range of greatest risk $(\geq 30 \%)$, because this is a criterion that increases risk in all individuals.

High levels of HDL-C have contributed to reducing the risk of coronary artery disease among men from 30 years onwards, and this difference was less significant among women.

Both in men and women, the presence of diabetes mellitus has made little contribution to increasing the score. The difference between the score expected and the mean score observed in diabetic individuals began to appear in men aged 60 years and above and in women aged 45 years and above, but the contribution towards increasing that difference was not significant.

Most adults (30-59 years) were among the individuals at the lowest risk of developing the disease, ie, a risk lower than 5\% (fig. 4). Among men, 118 adults were in that range of risk, representing $51.8 \%$ of the individuals in that age group in the sample studied. Among women, this prevalence was even greater, 243 women were in that range of risk, representing $76.2 \%$ of the women aged 30 to 59 years.

In both sexes, the elderly had a mean risk of developing coronary artery disease ranging from $10 \%$ to $19 \%$ as follows: 160 men, $34.7 \%$ being elderly, and 329 women, $46.7 \%$ being elderly, were in that range of risk; 142 (30.8\%) elderly men had a risk for coronary artery disease ranging from 20 to $29 \%$, while $114(16.2 \%)$ women were in that same range of risk.

A high proportion of elderly men $(25.2 \%)$ was classified in the highest-risk group, ie, risk for developing coronary artery disease $=30 \%$. This proportion among women was much lower $(4.8 \%)$. 
The medians of the risk score for developing coronary artery disease did not differ in each age group and sex according to familial income in minimum wages. When schooling, in years of study, was compared, the mean global score of the population whose schooling was less than 4 years was greater than that of the individuals whose schooling was greater than 4 years in adults $(\mathrm{P}<0.001)$, but not in the elderly. No significant difference was observed in the individuals studied in regard to the presence of $T$. cruzi infection (tab. II).

\section{Discussion}

The results of this study reveal that the probability of developing coronary artery disease within 10 years among men gradually increased as age advanced, reaching its peak $(25.1 \%)$ in the oldest age group (70-74 years). Among women, this phenomenon also occurred, but the greater mean risk $(13 \%)$ was slightly over half that observed among men. The increased risk of coronary artery disease with the increase in age occurred due to a combination of factors. According to Kannel and Wilson ${ }^{7}$, the incidence of cardiovascular diseases in adults doubles approximately at each decade of life, even in individuals with an excellent cardiovascular risk profile. Concomitantly, the increase in longevity enables longer periods of exposure to risk factors of cardiovascular diseases, which results in a greater probability of having the clinical manifestation of these diseases.

In Bambuí, men had higher risk scores and were more concentrated in the highest positions of the ranges of coronary risk than women did. This phenomenon has already been well reported, mainly in industrialized countries. The explanations for this difference are related to biological (sex) and cultural features and lifestyle (gender). The biological explanation for this fact could be the female protection provided by estrogen, which has a direct effect on the circulatory system, causing vasodilation and inhibiting the progression of the atherosclerotic processes, avoiding, therefore, ischemic events ${ }^{18}$. The explanations favoring gender emphasize the differences in the concentration of risk factors and in the manner by which men and women are exposed to these factors throughout life. The lower exposure to these risk factors experienced by women has a lower impact on the development of cardiovascular diseases in the medium and long run ${ }^{19}$.

According to the results obtained, a maximum potential reduction in coronary risk can be calculated by the percentage of reduction in the risk observed to the plateau of the risk expected according to age and sex. For example, in the elderly aged 70 or above, who have the greatest risks, prevention of the modifiable risk factors would mean a reduction of approximately $44 \%$ (from $25 \%$ to $14 \%$ ) for men and of 38\% (from 13\% to 8\%) for women. In 1996, among the population above the age of 60 years in Brazil, 28,479 deaths occurred due to coronary artery disease ${ }^{11}$. A $30 \%$ reduction in the risk of coronary artery disease would have a significant impact on the amount of potentially avoidable diseases and deaths.

The identification of the greatest risk factors for cardiovascular disease using population-based studies and effective control strategies combined with community education and monitoring of the high-risk individuals have contributed to a substantial drop in mortality due to cardiovascular diseases in almost all developed countries. In recent decades, a drop in mortality due to coronary and cerebrovascular diseases occurred in the United States and Western

\begin{tabular}{|lcc|}
\hline \multicolumn{2}{|c|}{ Table II - Framingham global risk score for coronary artery disease according to sex, age group, schooling, familial income, and T. cruzi infection. } \\
Bambuí Project, 1997
\end{tabular}


Europe ${ }^{20-22}$. An approximate 50-60\% drop in mortality due to cardiovascular disease has also been reported in Australia and Japan ${ }^{2}$.

Any of the major risk factors for coronary artery disease, even alone, may cause a manifest disease if left untreated for many years ${ }^{8}$. However, several studies have shown that these factors tend to occur in combination, and that effective prevention can only be achieved through a global approach to the risk profile of individuals and populations ${ }^{7}$. In the present study, 3 factors contributed more decisively to the final risk of coronary artery disease: smoking, high total cholesterol and high blood pressure. The coexistence of these risk factors has already been reported in Bambuí with hypertensive individuals, who have a 6-fold higher chance of simultaneously having 3 or more risk factors for coronary artery disease after adjusting for age and $\operatorname{sex}{ }^{14}$.

Greater schooling, even considering the cut-off point of only 4 years of study, was associated with a better coronary risk profile in adults of both sexes, but not in the elderly. This tendency is consistent with the results obtained in other developed countries ${ }^{23}$, suggesting a cohort effect, ie, the adult generation in that community has had greater access to the relatively recent initiative of spreading the preventive measures for coronary artery disease. In addition, the adults with greater schooling may have had greater access to and better use of that information.

Despite all the enormous pressure of accelerated population aging on morbidity and mortality due to cardiovascular diseases, the prevalence of several risk factors for those diseases in developing countries tend to be lower than that observed in developed countries, providing a window of opportunity to early and effective control of the epidemic projected for developing countries ${ }^{2}$. In Brazil, coronary artery diseases have contributed to $29.6 \%$ of the deaths due to cardiovascular diseases from 1996 to 1999 , with a mean mortality rate of 46.4 deaths per 100,000 inhabitants/year ${ }^{10}$. As population aging is a recent phenomenon in Brazil, the development and consolidation of healthy life habits among the enormous contingent of young individuals could lead to a future decline in the rates of coronary artery diseases in the country. Therefore, this moment of demographic transition that the country currently experiences represents another window of opportunity for the development of effective preventive strategies for cardiovascular diseases that may minimize the estimated impact of aging on morbidity and mortality.

According to the literature consulted, this is the first representative population-based study in Brazil to apply this methodology to assess the impact of each risk factor in the global risk of coronary artery disease in a community. Our results have a great internal validity and indicate that a wide margin exists to reduce the incidence of coronary artery disease in the community studied. There is no reason to think that the risk profile observed in Bambuí is worse than that in other small urban communities in the country, which represent approximately $70 \%$ of Brazilian municipalities. Our results emphasize the importance of assessing risk factors for coronary artery disease in the population at large and not only among patients who already have evidence of the disease.

\section{Acknowledgments}

\section{We thank the Bambuí Project, FINEP, FIOCRUZ, and} CNPq.

\section{References}

1. Beaghole R, Saracci R, Panico S. Cardiovascular diseases: causes, surveillance and prevention. Int J Epidemiol 2001;30:1-4.

2. Reddy KS, Yusuf S. Emerging epidemic of cardiovascular disease in developing countries. Circulation 1998;97:596-601.

3. Lopez AD. Assessing the burden of mortality from cardiovascular diseases. World Health Stat Q 1993;46:91-6.

4. Murray CJL, Lopez AD. Mortality by cause for eight regions of the world: Global Burden of Disease Study. Lancet 1997;349:1269-76.

5. Murray CJL, Lopez AD. Alternative projections of mortality and disability by cause 1990-2020: Global Burden of Disease Study. Lancet 1997;349:1498-504.

6. World Health Organization. The World Health Report, 1998. Geneve: WHO, 1998.

7. Kannel WB, Wilson PW. Comparison of risk profiles for cardiovascular events: implications for prevention. Arc Intern Med 1997;42:39-66.

8. Grundy SM, Pasternak R, Greenland P, Smith S, Fuster V. Assessment of cardiovascular risk by use of multiple-risk-factor assessment equations: a statement for heatlhcare professionals from the American Heart Association and the American College of Cardiology. Circulation 1999;100:1481-92.

9. Anderson KM, Wilson PW, Odell PM, Kannel WB. An updated coronary risk profile: a statement for health professionals. Circulation 1991;83:356-62.

10. Almeida FF, Barreto SM, Couto BRGM, Starling CEF. Fatores preditores da mortalidade e de complicações per-operatórias graves em cirurgia de revascularização do miocárdio. Arq Bras Cardiol 2003;80:51-60.

11. Lima-Costa MF, Guerra HL, Barreto SM, Guimarães RM. Diagnóstico da situação de saúde da população idosa brasileira: um estudo da mortalidade e das internações hospitalares públicas. Inf Epidemiol SUS 2000;9:23-41.

12. Lima-CostaMF, UchoaE, Guerra HL, Firmo JO, Vidigal PG, BarretoSM. The Bambuí health and ageing study (BHAS): methodological approach for a population based prospective study of the elderly in Brazil. Rev Saúde Publ 2000;34:126-35.
13. Lima-Costa MF, Barreto SM, Guerra HL, Firmo JO, Uchoa E, Vidigal PG. Ageing with Trypanosoma cruzi infection in a community where the transmission has been interrupted: the Bambuí Health and Ageing Study (BHAS). Int J Epidemiol 2001;30:887-93.

14. Wilson PW, D'Agostino RB, Levy D, Belanger AM, Silbershatz H, Kannel WB Prediction of coronary heart disease using risk factor categories. Circulation, 1998; 97:1837-47.

15. Barreto SM, Passos VM, Firmo JO, Guerra HL, Vidigal PG, Lima-Costa MF. Hypertension and clustering of cardiovascular risk factors in a community in Southeast Brazil: the Bambuí Health and Ageing Study. Arq Bras Cardiol 2001; 77:576-81.

16. Kannel, WB. Cardiovascular risk factors in the elderly. Coron Artery Dis 1997;8:565-75

17. StataStatistical Software. Release 7.0College Station, Texas: Stata Corporation 2001

18. Mendelsohn ME, Karas RH. The protective effects of oestrogen on the cardiovascular system. N Engl J Med 1999;340:1801-11.

19. Lawlor DA, Ebrahim S, Davey Smith G. Sex matters: secular and geographical trends in sex differences in coronary heart disease mortality. BMJ 2001;323: 541-45

20. Thom TJ. Stroke mortality trends: an international perspective. Ann Epidemiol 1993;3:509-18

21. Thom TJ, Epstein FH. Heart disease, cancer, and stroke mortality trends and their interrelations: an international perspective. Circulation 1994;90:574-82.

22. Bonita R, Beaglehole R. The enigma of the decline in stroke deaths in the United States: the search for an explanation. Stroke 1996;27:370-2

23. Leino M, Raitakari OT, Porkka KV, Taimela S, Viikari JS. Associations of education with cardiovascular risk factors in young adults: the Cardiovascular Risk in Young Finns Study. Int J Epidemiol 1999;28:667-75. 